\title{
A PRODUÇÃO, DIFUSÃO E RECEPÇÃO DE CONTEÚDOS TELEJORNALÍSTICOS NA ERA DA CONVERGÊNCIA DIGITAL
}

\author{
Production, Diffusion and Reception \\ of Tv News in the Era of Digital Convergence \\ Producción, Difusión y Recepción de Contenidos \\ Informativos Televisivos en la Era de la Convergencia Digital
}

Carlos Canelas

Unidade de Investigação para o Desenvolvimento do Interior Instituto Politécnico da Guarda, Portugal carlos.canelas@ipg.pt

Jorge Ferraz de Abreu Universidade de Aveiro e CIC.Digital, Portugal jfa@ua.pt

Jacinto Godinho

Universidade Nova de Lisboa e CIC.Digital, Portugal jacintog@hotmail.com

\section{Resumo}

Neste artigo, tendo por base uma revisão bibliográfica, são expostas algumas apreciações acerca da produção, difusão e recepção de conteúdos informativos televisivos na era da convergência digital, procurando assinalar e analisar as principais transformações nestes campos. Na era da convergência digital, os processos de conceção, distribuição e consumo de conteúdos jornalísticos transmitidos através da televisão estão a ser redefinidos.

Palavras-chave: Conteúdos Jornalísticos. Convergência Digital. Televisão.

\begin{abstract}
In this paper, based on a literature review, we present some assessments on the production, diffusion and reception of television news in the era of digital convergence and try to analyze the main changes in these areas. In the era of digital convergence, design, distribution and consumption processes of news content transmitted through television are being redefined.
\end{abstract}

Key words: Digital Convergence. News. TV. 


\section{Resumen}

En este artículo, basado en una revisión de la literatura, se exponen algunas consideraciones sobre la producción, difusión y recepción de contenidos informativos televisivos en la era de la convergencia digital, buscando señalar y analizar los principales cambios en estos campos. En la era de la convergencia digital, los procesos de concepción, distribución y consumo de contenidos informativos transmitidos a través de la televisión se están redefiniendo.

Palabras clave: Contenidos Informativos. Convergencia Digital. Televisión.

\section{INTRODUÇÃO}

Nos últimos anos, a convergência digital tem vindo a exercer uma grande influência em todas as áreas dos media informativos, tendo provocado alterações significativas em todas as fases dos processos de produção, difusão e recepção de conteúdos jornalísticos (PAVLIK, 2000，2001; SALAVERRÍA，2003，2009; JENKINS，2004，2006; DEUZE，2008b; SALAVERRÍA e GARCÍA AVILÉS, 2008; CANAVILHAS, 2009a; GARCÍA AVILÉS, 2010; SALAVERRÍA et al., 2010).

Nesta conjuntura, a televisão, não só na sua qualidade de meio informativo, está a atravessar tempos de mudança. Tal como refere Francisco Rui Cádima (2008a, 2008b), o setor televisivo está a passar por uma fase de profundas reconfigurações, tanto no que diz respeito às dinâmicas de mercado como no que se refere aos consumos. Na atualidade, por exemplo, os conteúdos televisivos são difundidos, para além das formas convencionais (hertziana, cabo e satélite), através de multiplataformas digitais, como sejam a IPTV (Internet Protocol Television), a Web TV ou a Mobile TV (CARDOSO et al., 2011).

Assim, a televisão encontra-se disseminada por diversos espaços e aparelhos (televisores [televisor convencional; LCD; Plasma], computadores, telemóveis, smartphones, tablets, consolas de jogos, media-centers, entre outros equipamentos fixos e portáteis), podendo ser visionada, pelos telespetadores, a qualquer hora, em qualquer lugar e a partir de diversos suportes de receção de conteúdos televisivos (JENKINS, 2006; GARCÍA AVILÉS, 2007; CARDOSO et al., 2011). Por consequência, a própria experiência televisiva está a ser modificada (CARDOSO et al., 2011).

Ainda que a televisão esteja, nos últimos tempos, a perder alguma da sua audiência para os novos media, especialmente no que concerne às faixas etárias mais jovens (DEUZE, 2008b; CÁDIMA, 2011), o pequeno ecrã continua a ser o medium preferido da maior parte dos cidadãos (BRANDÃO, 2006, 2010; GARCÍA AVILÉS, 2007; TORRES, 2011), já que 
ver televisão continua a ser a única atividade partilhada por todas as classes sociais e por todos os grupos etários (WOLTON, 1999; OBERCOM, 2011). Aliás, visionar televisão é uma atividade quotidiana para quase a totalidade da população mundial (ABREU, 2007).

Então, tal como sublinha Dominique Wolton (1994), a televisão ocupa um lugar de destaque na vida das pessoas, quer pela informação quer pelo entretenimento que proporciona, constituindo-se como a principal janela aberta sobre o mundo. Neste seguimento, na opinião de Ignacio Ramonet (1999), a caixa que teve a capacidade de mudar o mundo não é apenas o principal medium de entretenimento, mas é igualmente o principal meio de informação. Perante isso, como acrescenta Pierre Bourdieu (2005: 10), a televisão assume-se como o central meio noticioso, tendo uma "espécie de monopólio sobre a formação dos cérebros de uma parte muito importante da população".

Apesar de crítico, Giovanni Sartori (2000) reconhece que o mérito particularmente consensual da televisão é o de informar, entendendo o termo informar como fornecimento de notícias. Nesta perspetiva, para Nelson Traquina (2000), existe um crescente recurso às notícias televisivas, por parte dos indivíduos, como principal fonte de informação. Para muitas pessoas, as notícias veiculadas pela televisão continuam a ser a principal e, por vezes, a única fonte de informação (OBERCOM, 2011).

Neste contexto, centrando esta problemática no telejornalismo, seguidamente são apresentadas algumas considerações sobre a produção, transmissão e recepção de conteúdos noticiosos na era da convergência digital, procurando identificar e discutir as principais mudanças nestes campos.

\section{DESENVOLVIMENTO}

\subsection{Produção de conteúdos informativos televisivos}

No jornalismo televisivo, os processos de produção de conteúdos noticiosos sofreram e continuam a sofrer diversas transformações devido à implementação das "redações digitais integradas" (POWELL, 1998; GARCÍA AVILÉS e LEÓN, 2002; GARCÍA AVILÉS et al., 2004; GARCÍA AVILÉS, 2006b, 2006c, 2007b; AGUILAR-GUTIÉRREZ e LÓPEZ-DESOLÍS, 2010). Esta expressão, como nos explica Peter Powell (1998), pode ser entendida como sistemas digitais baseados no armazenamento de informações (textos, gráficos, imagens, sons, ...) em servidores centrais, através dos quais os profissionais da informação, sobretudo os jornalistas, têm acesso, em simultâneo, a partir dos computadores 
disponibilizados nas redações, para serem usadas nos processos de produção noticiosa. Por meio dos mencionados sistemas, os jornalistas podem realizar, em algumas situações, todas as tarefas de concepção de conteúdos noticiosos a partir dos seus próprios computadores (BANDRÉS et al., 2000; GARCÍA AVILÉS, 2002; GARCÍA AVILÉS, 2007b).

Estes sistemas digitais começaram a ser instalados nas redações televisivas a partir da segunda metade da última década do século XX, encontrando-se já implementados num grande número de emissoras televisivas mundiais (GARCÍA AVILÉS e LEÓN, 2002; GARCÍA AVILÉS et al., 2004; GARCÍA AVILÉS, 2007b; SALAVERRÍA e NEGREDO, 2008).

Os sistemas digitais de produção e difusão de conteúdos informativos estão baseados em servidores de vídeo, também denominados por video servers, sendo considerados depósitos de material audiovisual, com grande capacidade de armazenamento mas limitada, pelo que a sua boa gestão poderá ser a diferença entre o sucesso e o fracasso de aplicação de uma tecnologia cara (HENRIQUES, 2002). A instalação de servidores de vídeo nas redações televisivas permitiu retirar parcialmente as cassetes de vídeo de circulação, permitindo otimizar os fluxos de trabalho (GARCÍA AVILÉS, 2006c; NYE, 2007; AUSTERBERRY, 2009, 2011). Assim sendo, no âmbito da produção noticiosa televisiva, a utilização de servidores de vídeo apresentam diversas vantagens.

Quando os repórteres de imagem usam câmaras de vídeo que registam o material audiovisual em bruto em discos magnéticos ou óticos, tal como o XDCAM, este material é "ingestado" no servidor de vídeo, ou seja, os diversos ficheiros de vídeo contidos nos discos magnéticos ou óticos são copiados, em poucos minutos, para um servidor de vídeo, ficando imediatamente disponíveis para serem usados na edição de vídeo.

Porém, no caso dos repórteres de imagem que ainda utilizam câmaras de vídeo que gravam o material audiovisual em bruto em cassetes de vídeo, tais como as cassetes BETACAM SX ou DVCAM, a cópia do material audiovisual em bruto para o servidor de vídeo é mais demorada, visto que, nesta situação, o processo de captura decorre em tempo real, dito de outra forma, se o material audiovisual em bruto captado pelo repórter de imagem tiver uma duração de 30 minutos, este processo demora os 30 minutos. Contudo, a partir do momento em que os ficheiros de vídeo estejam alojados no servidor de vídeo, o acesso às imagens e ao respetivo áudio, por quem vai editar em vídeo, pode ser feito de forma aleatória, isto é, tem acesso imediato a cada ponto desejado, facilitando a localização dos planos que se pretende 
utilizar na conceção de conteúdos noticiosos televisivos (CROCOMO, 2001; CROCOMO e LAGE, 2001; HENRIQUES, 2002; GRANT et al., 2010).

Outra grande vantagem do uso dos servidores de vídeo prende-se com a possibilidade de vários profissionais da informação televisiva, como sejam os editores de imagem e os jornalistas, poderem ter acesso simultaneamente ao(s) mesmo(s) ficheiro(s) de vídeo, quer isto dizer que mais do que um profissional pode estar a trabalhar, ao mesmo tempo, com as mesmas imagens e sons (GARCÍA AVILÉS e LEÓN, 2002; HENRIQUES, 2002; GARCÍA AVILÉS, 2006c, 2007b; PIVETA, 2010; AUSTERBERRY, 2011).

Tanto mais que, quando os servidores de vídeo estão ligados em rede, os profissionais da informação televisiva podem aceder aos servidores de vídeo de outras delegações e viceversa. $\mathrm{Na}$ altura em que se usavam apenas cassetes de vídeo, apenas um profissional é que tinha acesso ao material audiovisual em bruto (AUSTERBERRY, 2009). Para que outros profissionais tivessem acesso ao mesmo material audiovisual em bruto era necessário fazer cópias, o que era moroso, já que, tal como já foi referido, as cópias eram efetuadas em tempo real e, sem esquecer que, quando a cassete de vídeo original estava a ser copiada, esta não podia ser utilizada na edição de vídeo. Por outra parte, a cópia de cassete de vídeo para outra cassete de vídeo significava quase sempre perda de qualidade, quer de imagem quer de áudio (AUSTERBERRY, 2011). Para além do mais, o uso constante das cassetes de vídeo degradava a fita, assim como os equipamentos de reprodução e gravação de cassetes de vídeo, ao fim de inúmeras horas de funcionamento.

Por outro lado, devido ao uso de servidores de vídeo, os jornalistas, a partir dos computadores que têm disponíveis na redação para editar em vídeo conteúdos informativos televisivos, têm acesso de imediato aos feeds enviados pelas agências noticiosas nacionais e internacionais (GARCÍA AVILÉS e LEÓN, 2002; MARTINS, 2005; GARCÍA AVILÉS, 2006d), podendo de imediato editar em vídeo os seus conteúdos noticiosos televisivos.

$\mathrm{O}$ acesso ao material de arquivo também é mais fácil, graças aos metadados inseridos nos ficheiros de vídeo, e, consequentemente, a sua recuperação, por parte dos profissionais da informação, é simples e, sobretudo, menos demorada (CROCOMO, 2001; GARCÍA AVILÉS et al., 2004; MARTINS, 2005; GARCÍA AVILÉS, 2006d).

Em termos de supervisão, os coordenadores e/ou os editores podem ter acesso, em qualquer momento, aos conteúdos noticiosos televisivos sobre os quais os jornalistas estão a trabalhar, de forma a acompanhar o desenvolvimento da sua construção e de modo a detetar possíveis erros antes dos conteúdos informativos televisivos serem transmitidos (GARCÍA 
AVILÉS e LEÓN, 2002; GARCÍA AVILÉS et al., 2004; GARCÍA AVILÉS, 2006c; SILCOCK, 2007).

A interligação dos servidores de vídeo de produção aos de emissão evita a maçadora operação de cópia para cassetes e respetivo transporte manual até à régie de produção, onde está a ser realizado o programa informativo, e posteriormente passagem à régie de continuidade de emissão (CROCOMO, 2001; HENRIQUES, 2002; GARCÍA AVILÉS et al., 2004; GARCÍA AVILÉS, 2006d; BONI, 2010). Além do mais, tal como refere Fernando Crocomo (2001), com a utilização dos servidores de vídeo, diminuem as probabilidades de erro como o posicionamento inicial dos conteúdos jornalísticos televisivos.

Os custos operacionais dos servidores de vídeo são mais baixos do que o uso de inúmeros equipamentos de vídeo, dado que o sistema está automatizado, necessitando de poucos recursos humanos para funcionar (NYE, 2007; AUSTERBERRY, 2009; GRANT et $a l ., 2010)$.

Devido aos servidores de vídeo e à ligação em rede, os jornalistas podem realizar diversas tarefas a partir de um único posto de trabalho, isto é, através de um computador, tais como: investigar recorrendo à Internet; visionar o material audiovisual em bruto captado pelo repórter de imagem e/ou enviado por agências noticiosas; elaborar o texto noticioso, que irá dar origem à voz-off, num processador de texto; montar o conteúdo informativo televisivo num editor de vídeo; entre outras tarefas (NYE, 2007).

A priori, as "redações digitais integradas" permitem uma produção mais ágil e necessitam de menos recursos humanos, contribuindo, desta maneira, para a redução dos custos de produção (GARCÍA AVILÉS e LEÓN, 2002). Por outra parte, possibilitam aos coordenadores e aos editores uma melhor supervisão do trabalho dos seus subordinados, podendo verificar em qualquer momento o ponto de situação de cada conteúdo noticioso (GARCÍA AVILÉS et al., 2004).

Contudo, a tecnologia digital por si só não garante uma melhor qualidade do produto jornalístico. Portanto, a tecnologia não deve controlar a capacidade produtiva e nem sequer deve ser encarada como o fator determinante das transformações verificadas na atividade jornalística (GARCÍA AVILÉS, 2007b). Tal como sublinha João Canavilhas (2009a: 51), «olhar para esta tecnologia na sua perspectiva meramente instrumental é reduzir drasticamente o seu potencial», acrescentando «mais do que o "como funciona?", interessa saber "para que serve?", "porque se utiliza?” e "em que circunstâncias a sua utilização é a melhor opção"» (CANAVILHAS, 2009a: 51). 
Para José Alberto García Avilés (2007b), o principal fator das mudanças no campo do jornalismo não é a tecnologia, mas sim fatores sociais, humanos e económicos. Ainda assim, John Pavlik (2000), Niina Rintala e Sanna Suolanen (2005) sublinham que a tecnologia digital influenciou e continuará a influenciar as diversas áreas do jornalismo. Todavia, como faz questão de lembrar García Avilés (2006b), os operadores televisivos não são empresas de tecnologias, mas organizações que produzem e emitem conteúdos. Nesta ótica, a tecnologia está ao serviço dos conteúdos e não o inverso.

No entanto, se a tecnologia digital for usada de uma forma adequada pelos profissionais da informação noticiosa, esta ajuda a melhorar a produtividade e a rentabilidade dos recursos, quer os humanos quer os técnicos (GARCÍA AVILÉS, 2007b). Por outro lado, a tecnologia digital pode facilitar o trabalho do jornalista televisivo, na medida em que as decisões sobre o teor dos diversos conteúdos noticiosos podem ser tomadas mais perto do início da emissão dos inúmeros programas jornalísticos e, caso seja necessário, é mais fácil e mais rápido introduzir modificações de última hora (PAVLIK, 2000; DEUZE, 2004; GARCÍA AVILÉS, 2007b).

Uma das consequências apontadas à digitalização das redações televisivas é que os jornalistas começaram a desempenhar outras funções/ tarefas que anteriormente eram executadas por profissionais altamente especializados, como seja a edição de imagem (COTTLE e ASHTON, 1999; GARCÍA AVILÉS, 2002, 2006a, 2006b, 2007b; GARCÍA AVILÉS e LEÓN, 2002; HEMMINGWAY, 2005; RINTALA e SUOLANEN, 2005; SALAVERRÍA e GARCÍA AVILÉS, 2008; SCOLARI et al., 2008; MICÓ et al., 2009; AGUDO e PALOMO TORRES, 2010; AGUILAR-GUTIÉRREZ e LÓPEZ-DE-SOLÍS, 2010; PIVETA, 2010, SOUZA e PIVETA, 2011a, 2011b, 2011c).

Por outra parte, também surgiram novas funções, como sejam o media manager ou o system manager, ligadas à operacionalização e manutenção dos sistemas digitais de produção e de difusão de conteúdos noticiosos (GARCÍA AVILÉS, 2006d; AGUILAR-GUTIÉRREZ e LÓPEZ-DE-SOLÍS, 2010). O media manager é o profissional que gere os conteúdos e administra os servidores de vídeo de produção e de emissão (AGUILAR-GUTIÉRREZ e LÓPEZ-DE-SOLÍS, 2010), enquanto o system manager tem a responsabilidade de garantir o bom funcionamento técnico do sistema (GARCÍA AVILÉS, 2006d).

Com o surgimento dos operadores televisivos privados e, posteriormente, com a proliferação dos canais temáticos noticiosos (emitindo 24 horas de informação televisiva por dia) e, sem esquecer, a concorrência dos novos media, sobretudo os disponibilizados na web, 
a produção jornalística noticiosa televisiva aumentou consideravelmente nos últimos tempos. Deste modo, os media noticiosos televisivos veem-se obrigados a emitir conteúdos jornalísticos em intervalos temporais cada vez mais curtos.

Esta constatação obriga a que, por um lado, os jornalistas televisivos aumentem a sua produção informativa e, por outro, diminuam o tempo dispensado na conceção de cada conteúdo jornalístico, sem que haja perda aparente de qualidade no produto noticioso (GARCÍA AVILÉS e LÉON, 2002; GARCÍA AVILÉS, 2006c). Assim, as redações televisivas optaram por implementar as "redações digitais integradas" com o propósito de produzir mais conteúdos noticiosos, encurtando o tempo de conceção, ao menor custo possível (GARCÍA AVILÉS, 2006c).

Para além da revolução tecnológica, as redações televisivas foram reorganizadas, quer no que toca às suas estruturas físicas, como a organização do espaço, quer no que respeita à redistribuição dos seus profissionais por este espaço (GARCÍA AVILÉS, 2006b; SALAVERRÍA e GARCÍA AVILÉS, 2008; MEIER, 2007, 2010; VERWEIJ, 2009). As paredes entre secções foram retiradas para tornarem as redações amplos espaços abertos, com o propósito de melhorar as condições de trabalho, permitindo facilitar a comunicação entre os profissionais da informação e os fluxos de trabalho e a colaboração entre meios (televisão, online, rádio e jornal) (GARCÍA AVILÉS, 2006b; MEIER, 2007; VERWEIJ, 2009). Várias classes e hierarquias profissionais laboram no mesmo espaço físico, como sejam: coordenadores; editores executivos; produtores; jornalistas; editores de imagem; infografistas; ... (MEIER, 2007; VERWEIJ, 2009). Com este novo conceito de redação pretende-se fomentar um novo modelo de organização e uma nova forma de pensar e atuar em termos jornalísticos (MEIER, 2010).

\subsection{Disseminação e recepção de conteúdos informativos televisivos}

As redações televisivas continuam a produzir os conteúdos para serem difundidos pelas formas tradicionais (hertziana, cabo e/ou satélite) e, por consequência, para serem consumidos da maneira habitual, ou seja, para serem visionados pelos telespectadores através de televisores, dado que ainda é aí que concentram o maior número de audiência.

Porém, nos últimos anos, os conteúdos noticiosos televisivos não são apenas consumidos pelos telespectadores pelo método tradicional, ou seja, pelo televisor. Isto é, os telespectadores podem aceder às emissões e/ou aos conteúdos televisivos a partir de diversos dispositivos de recepção, tais como: computadores; telemóveis; smartphones; tablets; 
consolas de jogos; media-centers; entre outros equipamentos fixos e portáteis. Daí que, atualmente, não é mais necessário um televisor para se ver televisão (CARDOSO et al., 2011). Como é evidenciado por Gustavo Cardoso, Jorge Vieira e Sandro Mendonça (2011), verifica-se a independência do consumo de televisão face à disponibilidade do televisor, permitindo aos telespectadores cada vez mais consumirem, onde e quando quiserem, conteúdos de acesso em plataformas com grande capacidade de armazenamento (Youtube, Vimeo), usando aparelhos leves e pessoais, mas com ecrãs relativamente grandes em proporção ao seu tamanho (smartphones e tablets).

No contexto da distribuição dos conteúdos noticiosos produzidos pelas redações televisivas, após a sua transmissão via tradicional, estes são disponibilizados noutras plataformas. Por norma, nos websites oficiais das estações de televisão existe uma secção onde os cibernautas podem aceder aos conteúdos informativos. Nos últimos anos, a quantidade de conteúdos noticiosos disponibilizados pelos media informativos, nos seus websites, aumentou consideravelmente (MICÓ e MASIP, 2008), e os utilizadores da web valorizam o seu uso na informação jornalística (CANAVILHAS, 2009b).

Nos primeiros tempos, os operadores televisivos limitavam-se a estar presente na web através de uma simples página (LERMA, 2011), pois os seus responsáveis não viam com bons olhos a disponibilização dos seus conteúdos em ambiente online, temendo que esta prática pudesse contribuir para a perda de audiência (MESO AYERDI e LARRONDO URETA, 2011). Nessa altura, as televisões usavam a web para expor informação, geralmente em suporte escrito, que complementasse os programas televisivos transmitidos na emissão tradicional. Mais tarde, o pequeno ecrã considerou a web como uma mera plataforma de difusão de conteúdos audiovisuais, encarando-a como uma estratégia de expansão (MESO AYERDI e LARRONDO URETA, 2011). Os operadores televisivos ainda não se tinham apercebido das mudanças que se estavam a verificar na forma de se consumir os conteúdos televisivos e, sobretudo, os novos hábitos da audiência, principalmente dos mais jovens.

$\mathrm{Na}$ verdade, a web proporcionou o surgimento de novas formas de distribuição e, consequentemente, de recepção de conteúdos audiovisuais. Perante esta realidade, os operadores de televisão facultam o acesso, nos respetivos websites e nas aplicações para os dispositivos móveis (smartphones e tablets), aos conteúdos noticiosos veiculados na emissão televisiva tradicional.

No entanto, na maior parte das vezes, estes conteúdos não foram sujeitos a qualquer tipo de tratamento com o propósito de adaptá-los ao meio online (CANAVILHAS, 2008, 
2009b; LOUREIRO, 2008; MICÓ e MASIP, 2008; CANELAS, 2012). Deste modo, como lembra João Canavilhas (2009b), nestes casos, não se pode falar de uma linguagem nova, pois os conteúdos oferecidos aos cibernautas são os mesmos que foram difundidos na emissão tradicional, mantendo a linguagem jornalística e televisiva original. De qualquer maneira, com esta prática, os operadores de televisão dão a possibilidade a qualquer pessoa de ver ou rever os seus noticiários, em qualquer momento, em qualquer lugar e através de diversos suportes de recepção.

Por outro lado, a televisão de hoje, designadamente a disponibilizada pelos serviços de televisão paga, oferece novas funcionalidades, como sejam: Timewarp, funcionalidade baseada na tecnologia Advanced Personal Cloud Recordings, permite aos telespectadores recuperar as emissões já transmitidas relativas a um certo período de tempo; Restart TV, que dá a oportunidade aos telespectadores, mesmo que o programa que pretendem ver já tenha iniciado, de poderem começar a visioná-lo do início; Pausa Tv, que possibilita aos telespectadores parar a emissão e retomá-la, mais tarde, do ponto em que a transmissão foi interrompida; gravação de vários programas em simultâneo, entre outras funcionalidades. As funcionalidades destacadas libertam os telespectadores da tirania dos alinhamentos impostos pela televisão tradicional.

Face ao exposto, a efetivação do serviço de televisão nunca foi tão independente das variáveis tempo e espaço em que o consumo tem lugar (CARDOSO et al., 2011). De acordo com Gustavo Cardoso, Jorge Vieira e Sandro Mendonça (2011), «a experiência televisiva é pensada, cada vez mais, pelos consumidores em função da possibilidade de atingirem uma navegação em rede e se moverem, sem obstáculos, pelas diversas plataformas - conforme a sua conveniência ou preferência». Na mesma linha de raciocínio, Francisco Rui Cádima (2011: 9) escreve que «a televisão será cada vez mais um híbrido pós-televisivo, emergente através de um novo conceito e interface ubíquo de multimédia interactivo, em diferentes formatos e suportes, um mero ecrã nómada, como uma espécie de nova extensão da mente».

Assim, assiste-se a uma maior possibilidade de libertação dos telespectadores face aos conteúdos pré-programados e temporalidades impostas pela lógica centrada na oferta das estações (CARDOSO et al., 2011). 


\section{CONSIDERAÇÕES}

Este artigo, sustentado por uma revisão bibliográfica, procurou expor algumas considerações sobre a produção, a veiculação e a recepção de conteúdos noticiosos televisivos na era da convergência digital.

\section{REFERÊNCIAS}

ABREU, Jorge T. Ferraz. Design de Serviços e Interfaces num Contexto de Televisão Interactiva. Tese de Doutoramento. Aveiro: Universidade de Aveiro, 2007.

AGUDO, Juan Miguel; PALOMO TORRES, Bela. Convergencia y nuevas rutinas profesionales: luces y sombras del periodista polivalentes en las redacciones españolas. In: LÓPEZ GARCIA, Xosé; PEREIRA FARIÑA, Xosé. (Coords.). Convergencia Digital: reconfiguración de los medios de comunicación en España. Santiago de Compostela: Universidade de Santiago de Compostela, p. 129$148,2010$.

AGUILAR-GUTIÉRREZ, Manuel; LÓPEZ-DE-SOLÍS, Iris. Nuevos modos de trabajo de una redacción digital integrada: el caso de los servicios informativos de TVE. El profesional de la información, v. 19, n. 4, p. 395-403, 2010.

AUSTERBERRY, David. Studio Production Recording. Broadcast Engineering, 2009.

AUSTERBERRY, David. Losing VTRs. Broadcast Engineering, 2011.

BANDRÉS, Elena; GARCÍA AVILÉS, José Alberto; PÉREZ, Gabriel; PÉREZ, Javier. El periodismo en la televisión digital. Barcelona: Paidós, 2000.

BONI, Fernanda Aiex. O Aparelho e a Representação do Real na Edição de Imagens no Telejornalismo. Dissertação de Mestrado. Brasil: Universidade Estadual Londrina, 2010.

BOURDIEU, Pierre. Sobre Televisão. Oeiras: Celta Editora, 2005.

BRANDÃO, Nuno Goulart. PRIME TIME: do que falam as notícias dos telejornais. Lisboa: Casa das Letras, 2006.

BRANDÃO, Nuno Goulart. As Notícias nos Telejornais: que serviço público para o século XXI. Lisboa: Editora Guerra \& Paz, 2010.

CÁDIMA, Francisco Rui. A Crise do Audiovisual Europeu: 20 anos de Políticas Europeias. Lisboa: Media XXI, 2008a.

CÁDIMA, Francisco Rui. Do Audiovisual Europeu à Europa dos Cidadãos. Revista de Economía Política de las Tecnologías de la Información y Comunicación, v. 10, n. 3, 2008 b.

CÁDIMA, Francisco Rui. A Televisão, o Digital e a Cultura Participativa. Lisboa: Media XXI, 2011. 
CANAVILHAS, João. Webnotícia: propuesta de modelo periodístico para la www. Covilhã: LabCom, 2008.

CANAVILHAS, João. Ensino do Jornalismo: o digital como oportunidade. In: FIDALGO, Joaquim e MARINHO, Sandra. (Orgs.). Actas do Seminário "Jornalismo: mudanças na profissão, mudanças na formação”. Braga: Centro de Estudos de Comunicação e Sociedade da Universidade do Minho, p. 49-56, 2009a.

CANAVILHAS, João. Los efectos del vídeo en la noticia Web. Trípodos, n. 35, p. 147-161, 2009b.

CANELAS, Carlos. O Uso do Vídeo pelos Operadores Generalistas Televisivos Portugueses na Disseminação de Conteúdos Noticiosos através da Web. In: BASTOS, Helder e ZAMITH, Fernando. (Orgs.). Ciberjornalismo: modelos de negócio e redes sociais, Porto: Edições Afrontamento, p. 5567, 2012.

CARDOSO, Gustavo; VIEIRA, Jorge; MENDONÇA, Sandro. Ecrãs em Rede: Televisão. Lisboa: OberCom, 2011.

COTTLE, Simon; ASHTON, Mark. From BBC Newsroom to BBC Newscentre: on changing technology and journalist practices. Convergence: The International Journal of Research into New Media Technologies, v. 5, n. 3, p. 22-43, 1999.

CROCOMO, Fernando Antonio. O Uso da Edição Não-linear Digital: as novas rotinas no telejornalismo e a democratização de acesso à produção de vídeo. Dissertação de Mestrado. Brasil: Universidade Federal de Santa Catarina, 2001.

CROCOMO, Fernando Antonio; LAGE, Nilson. O Uso da Edição Não-linear Digital no Telejornalismo Diário de uma Emissora de TV e as Novas Rotinas de Produção. INTERCOM Sociedade Brasileira de Estudos Interdisciplinares da Comunicação, 2001.

DEUZE, Mark. What is Multimedia Journalism?. Journalism Studies, v. 5, n. 2, p. 139-152, 2004.

DEUZE, Mark. The Changing Context of News Work: Liquid Journalism and Monitorial Citizenship. International Journal of Communication, v. 2, p. 848-865, $2008 \mathrm{a}$.

DEUZE, Mark. The Professional Identity of Journalists in the Context of Converge Culture.

Observatorio Journal, n. 7, p. 103-117, 2008b.

GARCÍA AVILÉS, José Alberto. Periodismo polivalente y convergente: riesgos y oportunidades para el periodismo audiovisual. CHASQUI: Centro Internacional de Estudios Superiores de Comunicación para América Latina, n. 79, p. 38-43, 2002.

GARCÍA AVILÉS, José Alberto. Convergencia y polivalencia periodística en televisiones europeas y norteamericanas. Global Media Journal, 2006a.

GARCÍA AVILÉS, José Alberto. Desmitificando la convergencia periodística. CHASQUI: Centro Internacional de Estudios Superiores de Comunicación para América Latina, p. 34-39, 2006b.

GARCÍA AVILÉS, José Alberto. El periodismo audiovisual ante la convergencia digital. Espanha: Universidad Hernández, 2006c.

GARCÍA AVILÉS, José Alberto. Las redacciones de los canales 'todo noticias` como laboratorio de periodístico: los casos de BBC News 24 y Rainews 24. Trípodos, n. 19, p. 83-97, 2006 d. 
GARCÍA AVILÉS, José Alberto. El infoentretenimiento en los informativos líderes de audiencia en la Unión Europea. Análisis, n. 35, p. 47-63, 2007.

GARCÍA AVILÉS, José Alberto. Nuevas tecnologías en el periodismo audiovisual. Revista de la Faculdad de Ciencias Sociales y Jurídicas de Elche, v. 1, n. 2, p. 59-75, 2007.

GARCÍA AVILÉS, José Alberto. Convergencia en noticias Cuatro y CNN+: una transición incompleta. In: LÓPEZ GARCIA, Xosé e PEREIRA FARIÑA, Xosé. (Coords.). Convergencia Digital: reconfiguración de los medios de comunicación en España, Santiago de Compostela: Universidade de Santiago de Compostela, p. 213-222, 2010.

GARCÍA AVILÉS, José Alberto; LEÓN, Bienvenido. Journalistic Practice in Digital Televisión Newsrooms: the case of Spain's Tele 5 and Antena 3. Journalism, v. 3, n. 3, p. 355-371, 2002.

GARCÍA AVILÉS, José Alberto; LEÓN, Bienvenido; SANDERS, Karen; HARRISON, Jackie. Journalists at digital television newsrooms in Britain and Spain: workflow and multi-skilling in a competitive environment. Journalism Studies, v. 5, n. 1, p. 87-100, 2004.

GRANT, August E.; BROWN, Tim; NACHLINGER, Harvie. Accelerating Technologies: The Diffusion of Nonlinear Video Editing. Electronic News, v. 4, n. 4, p. 188-202, 2010.

HEMMINGWAY, Emma. PDP, The News Production Network and the Transformation of News. Converge, v. 11, n. 3, p. 8-27, 2005.

HENRIQUES, Carlos Alberto. A escola digital, ou o ensino na ponta dos dedos. In: MIRANDA, José A. Bragança e SILVEIRA, Joel Frederico. (Orgs.). As Ciências da Comunicação na viragem do Século, Actas do I Congresso da Associação Portuguesa de Ciências da Comunicação. Lisboa: Veja, p. 174-179, 2002.

JENKINS, Henry. The Cultural Logic of Media Convergence. International Journal of Cultural Studies, v. 7, n. 1, p. 33-43, 2004.

JENKINS, Henry. Convergence Culture: where old and new media collide. Nova Iorque: New York University, 2006.

KUKLINSKI, Hugo Pardo. El periodista polivalente: transformaciones en el perfil del periodista a partir de la digitalización de los medios audiovisuales catalanes. Zer, v. 13, n. 25, p. 37-60, 2008.

LERMA, Antonio. Convergencia de la televisión con Internet. Estudio de la televisión y su implantación en la Web. In: ORTEGA, Félix e CARDEÑOSA, Laura. (Eds.). Nuevos medios, nueva comunicación: libro de actas do II Congreso Internacional Comunicación 3.0. Salamanca: Universidade de Salamanca, p. 126-148, 2011.

LOUREIRO, Luís. A notícia de televisão na Internet: o fim do alinhamento, o princípio de quê?. In: Actas do 5. ${ }^{\circ}$ Congresso da Associação Portuguesa de Ciências da Comunicação. Universidade do Minho, Braga, 2007.

MARTINS, Ana Isabel Costa. Da Caixa Mágica à Caixa de Pandora - Repensar os Poderes e os Limites da TV. O Caso da SIC Notícias. In: Atas do 4. ${ }^{\circ}$ Congresso da Associação Portuguesa de Ciências das Comunicação, 2005.

MEIER, Klaus. Innovations in Central European Newsrooms: Overview and case study. Journalism Practice, v. 1, n. 1, p. 4-19, 2007. 


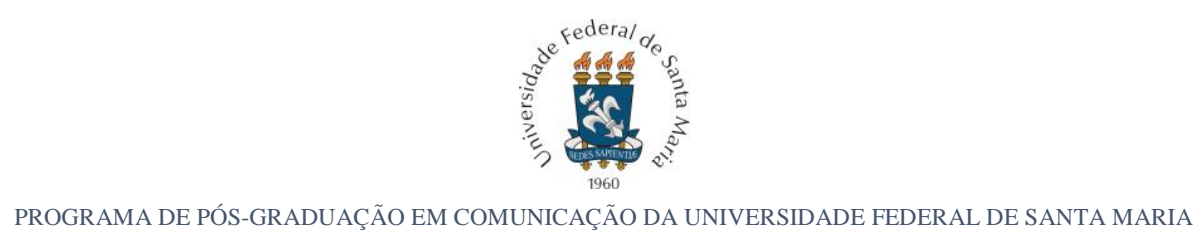

MEIER, Klaus. El periodismo en cambio. Digitalización y convergencia de redacciones en Alemania. Revista Comunicación y Ciudadanía, n. 3, p. 44-53, 2010.

MESO AYERDI, Koldobika; LARRONDO URETA, Ainara. Cambios en las estrategias de las audiencias de las páginas web de televisión. Análisis de la participación en los públicos autonómicos en España. In: ORTEGA, Félix e CARDEÑOSA, Laura. (Eds.). Nuevos medios, nueva comunicación: libro de actas do II Congreso Internacional Comunicación 3.0, Salamanca: Universidade de Salamanca, p. 53-68, 2011.

MICÓ, Josep; MASIP, Pere. Recursos multimedia en los cibermedios españoles. Análisis del uso del vídeo en El País.com, El Mundo.es, La Vanguarda.es y Libertad Digital. Trípodos, n. 23, p. 89-105, 2008.

MICÓ, Josep; MASIP, Pere; BARBOSA, Suzana. Models of Business Convergence in the Information Industry. Sociedade Brasileira de Pesquisa em Jornalismo, 2009. Disponível em: <www.unb.br/ojsdpp/include/getdoc.php?id=684...220...pdf >, Acesso em: 4 nov. 2015.

NYE, Chad Flynn. Diffusion of Nonlinear Editing Systems in U.S. Local Television. Dissertação de Mestrado. EUA: Universidade de Oklahoma, 2007.

OBERCOM. A Televisão na Sociedade em Rede. Lisboa: OberCom, 2011.

PAVLIK, John. The Impact of Technology on Journalism. Journalism Studies, v. 1, n. 2, p. 229-237, 2000.

PAVLIK, John. Journalism and New Media. Nova Iorque: Columbia University Press, 2001.

PIVETA, Patrícia. A Evolução Tecnológica como Interferência na Linguagem Televisual: o percurso da edição no telejornalismo da TV Coroados. Dissertação de Mestrado. Brasil: Universidade Estatual de Londrina, 2010.

POWELL, Peter. New Direction for the Integrated Newsroom. TVB Europe, v. 7, n. 9, 1998.

RAMONET, Ignacio. A Tirania da Comunicação. Porto: Campo das Letras, 1999.

RINTALA, Niina; SUOLANEN, Sanna. The Implications of Digitalization for Job Descriptions, Competencies and the Quality of Working Life. Nordicom Review, v. 26, n. 2, p. 53-67, 2005.

SALAVERRÍA, Ramón. Convergencia de medios. CHASQUI: Centro Internacional de Estudios Superiores de Comunicación para América Latina, n. 81, p. 32-39, 2003.

SALAVERRÍA, Ramón; GARCÍA AVILÉS, José Alberto. La convergencia tecnológica en los medios de comunicación: retos para el periodismo. Trípodos, n. 23, p. 31-47, 2008.

SALAVERRÍA, Ramón; NEGREDO, Samuel. Periodismo integrado: convergencia de medios y reorganización de redacciones. Barcelona: Sol90Media, 2008.

SALAVERRÍA, Ramón. Los medios de comunicación ante la convergencia digital. Navarra: Depósito Académico Digital Universidad de Navarra, 2009.

SALAVERRÍA, Ramón; GARCÍA AVILÉS, José Alberto; MASIP, Pere. Concepto de convergencia periodística. In: LÓPEZ GARCIA, Xosé e PEREIRA FARIÑA, Xosé. (Coords.). Convergencia Digital: reconfiguración de los medios de comunicación en España. Santiago de Compostela: Universidade de Santiago de Compostela, p.41-64, 2010. 
SARTORI, Giovinni. Homo Videns: televisão e pós-pensamento. Lisboa: Terramar, 2000.

SCOLARI, Carlos Alberto; MICÓ SANZ, José Lluís; GUERE, Héctor Navarro;

SILCOCK, B. William. Every edit tells a story Sound and the Visual Frame: a comparative analysis of videotape editor routines in global newsrooms. Visual Communication Quarterly, v. 14, n. 1, p. 315, 2007.

SOUZA, Florentina das Neves; PIVETA, Patrícia. A edição em TV na fase digital. In: Atas do VIII Encontro Nacional de História da Mídia, 2011a.

SOUZA, Florentina das Neves; PIVETA, Patrícia. A edição na reportagem telejornalística: um estudo comparativo do processo de edição linear e digital. In: Atas do XXIV Congresso Brasileiro de Ciências da Comunicação. INTERCOM, 2011b.

SOUZA, Florentina das Neves; PIVETA, Patrícia. A evolução tecnológica na edição do telejornalismo. FAMECOS, v. 18, n. 2, p. 431-445, 2011 c.

TORRES, Eduardo Cintra. A Televisão e o Serviço Público. Lisboa: Fundação Francisco Manuel dos Santos, 2011.

TRAQUINA, Nelson. Jornalismo 2000: o estudo das notícias no fim do século XX. Revista de Comunicação e Linguagens, n. 27, p. 15-31.

VERWEIJ, Peter. Making Convergence Work in the Newsroom: A Case Study of Convergence of Print, Radio, Television and Online Newsrooms at the African Media Matrix in South Africa During the National Arts Festival. Converge, v. 15, n. 1, p. 75-87, 2009.

WOLTON, Dominique. Elogio do Grande Público: uma teoria crítica da Televisão. Porto: Edições ASA, 1994.

WOLTON, Dominique. Pensar a Comunicação. Alvés: DIFEL, 1999. 
Original recebido em: 11 de abril de 2015

Aceito para publicação em: 24 de janeiro de 2017

\section{Carlos Canelas}

Professor Adjunto na Escola Superior de Educação, Comunicação e Desporto do Instituto Politécnico da Guarda, em Portugal. Em termos académicos, é Doutorado em Informação e Comunicação em Plataformas Digitais pelas Universidades de Aveiro e Porto.

Jorge Ferraz de Abreu

Professor Auxiliar no Departamento de Comunicação e Arte na Universidade de Aveiro, Portugal. Em termos académicos, é Doutorado em Ciências e Tecnologia da Comunicação pela Universidade de Aveiro.

\section{Jacinto Godinho}

Professor Auxiliar na Faculdade de Ciências Sociais e Humanas da Universidade Nova de Lisboa, Portugal. Em termos académicos, é Doutorado em Ciências da Comunicação pela Universidade Nova de Lisboa.

Esta obra está licenciada sob uma Licença Creative Commons. 